\title{
Exploring the Journals using Artificial Bee Colony algorithm
}

\author{
K. Radhika ${ }^{1}$, M. Sowndarya ${ }^{2}$, R. Suganya ${ }^{3}$, A. Vallikkannu ${ }^{4}$ \\ UG, Department of CSE, MKCE, Karur, India ${ }^{1,2,3,4}$
}

\begin{abstract}
The assessment of academic journals is a critical task to numerous scientific actions. These assessments may have an important influence on various issues reaching from publishing articles in academic journals to determining. The main aim is to develop the system that have the fair means of the selection of the best journal. Here we propose the system for ranking the journals in a huge data set which may include both similar and dissimilar distances of values from which the effective means of results being declared. A method for exploring the journals using, Artificial Bee Colony algorithm is presented to lessen the effort in working with the growth of data volumes. Artificial Bee Colony (ABC) is introduced for web mining for the both Journal Citation Reports and proposed data sets. This algorithm results us with relevant information for the questions posted by the clients when related to present Fuzzy c-means algorithm.
\end{abstract}

Keywords: Citation, Impact factor, Eigen factor, Artificial Bee colony.

\section{INTRODUCTION}

Journal ranking is presented as a research valuation tool in countries across the world. The existing Peer review and indicator methods are demoralized for the task in a struggle to afford measureable tools for evaluating academic journals. The data driven assessment measures has been established on citations, downloads and other features of journals, that is more benefits of being impartial, consuming few time and economics compared valuations which grounded on peer review by human experts.

The main aim is to develop the system that have the fair means of the selection of the best journal. Here we propose the system for ranking the journals in a huge data set which may include both similar and dissimilar distances of values from which the effective means of results being declared. Finally we need to select those who are efficient as well as have the qualification to obtain the results.

\section{DOMAIN INTRODUCTION}

Data mining known as knowledge discovery process, with various database technologies, deliver active way to resolve the occurring problem. Data mining is a computing process that relates artificial intelligence and the machine learning. It is accomplished by providing automated analysis of the unique data, creating inductive thinking, and mining theoretical information. As data mining can carry modest benefits and commercial profits to productions, a great amount of educational administrations and the IT corporations worldwide has thrown significant research. Numerous typical algorithms and software's have been advanced, and such practices can be considered according to altered criteria. These methods may be separated by two groups founded on the application:
Domain specific techniques and the common techniques. Classification: Approaches classically work in altering original human information and the criteria for measuring periodical potentials in rules, decision trees, and numerous methods of classifiers over knowledge from ranking outcomes specialists.

\section{LITERATURE SURVEY}

[1] "Peer Based Review" The Australian Research Council (ARC)

Newly, several countries has executed their national projects for theoretical productions assessment and reckonable charge, like the Research Excellence of Framework of United Kingdom and the Excellence Research for Australia (ERA). In this ways, attractive expert boards to achieve an inclusive peer review are perhaps the greatest dependable practice to make an impartial effect [2].

\section{(i) Research Excellence Framework in UK}

This framework is used for measuring the excellence of research in UK Education Institutions. The main purpose is to crop valuation outcomes for suggestion completed by them. The ending results of REF may have stimulus upon countless parts of research actions in UK. Theoretically, REF is a method of professional analysis. HEIs are requested to style proposals which are evaluated by experts, employed by the supervision of chief boards. The main conclusion of the valuation will be a total quality profile granted to each suggestion.

\section{(ii)Excellence Research for Australia}

The Excellence Research for Australia (ERA) is showed by them, determinations might include many excellences 
of researches conducted in Australian higher education institutions. The foremost results of ERA is the Ranking Journals List and out its complete ranking lists of higher to 20,000 journals in 2010, 2012, correspondingly. In excess of 700 specialists are complicated to help the ARC with emerging the journal grade information in which individual journal has a solitary excellence evaluation.

\section{[2] "How to evaluate journal impact} factors"J.Stegmann et al.

All year, the Thomson Reuters Journal Citation Reports ${ }^{\circledR}$ (JCR) studies the effect of all research journals. They discover the association among citing and cited journals, to discover the world's foremost journals [1].

\section{Journal Citation Reports}

The 2015 Journal Citation Statement revision which was unconfined in June 2016, held that IEEE journals remain to continue places at the topmost in their fields.

Impact Factor is calculating middling amount of articles that a journals printed in the previous two years has been cited in JCR years. This outcome is usually used and trusted upon by practical periodicals and their investors.

Eigenfactor Score computes the amount of periods that a journal available in prior five years has been cited in JCR years which is seeing which journals will have paid those citations. High-scoring journals will have a better inspiration in the scientific civic.

[3]"Problems with Fuzzy C-Means Clustering and the Similar Algorithms with the High Dimensional Data Sets"Roland Winkler, Frank Klawonn, Rudolf Kruse

The Fuzzy new c-means grouping and consequences of the fuzzy new c-means are fetching actual fruitful on many grouping difficulties. Fuzzy c-means clustering and algorithms that are connected to fuzzy clustering face difficulties with high dimensional data sets and a huge amount of prototypes. In that paper, they gave ideas about c-means, noisy clustering and the fuzzy new c-means with fuzzier task and it is like noise alternate. A distinct test data set is used to show weaknesses that is optimal for clustering algorithms of the high dimensions. It shows that a great amount of the various prototypes effects in the clustering process with a similar way as a great count of the dimension. Lastly, they showed the harmful things of great dimensional data groups may be minimized by altering algorithm's constraint, i.e. Fuzzier, liable on the amount of dimensions.

It consists numerous stimulating claims. For example clustering comparable music collections, web requests, and image credit or biochemical difficulties. Numerous tool now is not intended in holding the hundreds of new dimension, or that may be restored to grades of freedom. Maximum of the cluster methods effort moderately in little dimensions, yet particularly fuzzy c-means algorithm (FCM), appears to be failed in high dimensions. They provide vision into tricky and conduct of the FCM along with by products in large new dimensions [3].
[4]"Fused-Link: The Distance-Based Collection of the Journal Indicators"Navjot Kaur, Jaspreet Kaur, Navneet Kaur

The fused-link among the periodicals specifies relationship of the new impact of separate journals; the grades of the two journals with robust associations are possibly 'close' to others. The Ranking of the nearby neighbours offer further valuable data than the grades of their remote users. Distance-weighted method is distorted in that paper to categorize the positions of periodicals, where the elects of neighbours are weighted by the consequent link strength. The Ranked Journals List providing by the ERA have complicated a huge cluster of specialists to grade a huge amount of theoretical journals. Though debates bounded the final result, is worked by researchers as standard to associate journals ranking conclusions produced by automated mechanisms versus human expert's decisions.

[5]"The Artificial Bee Colony (Abc) Algorithm for the Ranking Web Pages"Anuradha G, Lavanya Devi G

The World Wide Web (WWW) is the fast upward technology on the all aspects and an immense, explosive and the data resource in world. The main target of Search engine is to service the most appropriate data to the users based to their requirements, generally search engine provide huge results for any user's request. To frontier the results information, it is key to allocate grade the web pages in a capable way. Artificial Bee Colony (ABC) is an innovative method that is used in resolving problems. This approach as a newest method for mining mostly in grading web leaves. It calculates the user interest, the total web site relation and evolution analysis rate which is used for handing over grade the web pages.

The tentative results shows high effectiveness of the future method when compare with the traditional ranking algorithms. Here we calculate the user interest and the growth analysis rate, and the total site references. The algorithm provides more related data for the requested asked by the user while compare with previous algorithm. They had new analysis achieved by seeing 5000url's. Aim of this algorithm is to rank for web pages based on the user's interest and the total sites linking and then the growth analysis rate [5].

\section{EXISTING SYSTEM}

In existing approaches they followed peer based method in which the judges rank the best journals. Then comes the report generation format for ranking the best journals based on various parameters such as impact factor that compares citations of journals to the ratio of the total number of articles published; total citations is the another factor which gives the information of the number of visits of a particular journal; then Eigen factor is a other kind of parameter which compares with last five years publications raises. Then next approach followed is the clustering algorithms which can be applied only for the 
pre-defined datasets and no extension of previous data sets will be provided. It also be applied only for the smaller datasets. Then ABC approach is applied for websites in which network traffic, total linking, page views and so on.

\section{DISADVANTAGES}

$>$ Results are not informative

$>$ Unreliable and inaccurate results

$>$ High time factor

$>$ Fail to work on large datasets

$>$ Difficult to apply on dissimilar data

\section{PROPOSED SYSTEM}

A method for exploring the journals using $\mathrm{ABC}$ algorithm is introduce to lessen the effort of selling the enlargement of data volumes. It is a new draw near as a new method for web mining for the both JCR and new data sets. This algorithm can be extended for special datasets. This algorithm eventually provides extra related data when compared to existing Fuzzy new c-means. It saves the times and produce the accurate solution and its efficiency and feasibility is high. In this $\mathrm{ABC}$ algorithm true and accepted outcome will be found out as it involves the both sets of data. They can be easily applied to work on the large datasets with large number of prototypes.

\section{EXPERIMENTAL RESULT}

\section{Modules:}

$>$ Retrieval of dataset from JCR

$>$ New dataset which includes the additional parameters introduced by $\mathrm{ABC}$ algorithm

$>$ Calculate efficient criteria for new dataset by using algorithm

$>$ Calculate the total efficiency by comparing both datasets and rank the journals which matches in common on analysing the various data sets like Journal Citation Report with additional prototypes we found that we can get efficient search analysis result. The sample outcome of our project is given below. This shows the journals in the ranking order based on the various factors like Impact factor, Total citation, Eigen factor with user interest rate, growth analysis rate and total reference

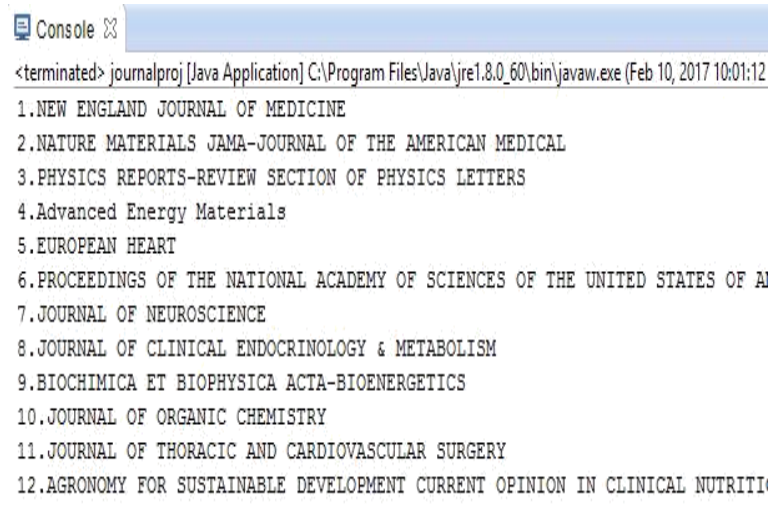

\section{CONCLUSION}

In this paper exploring of the best journal involves two different datasets such as JCR and human expert analysis. We have introduced a uniformity checking method, based on the $\mathrm{ABC}$ algorithm approach that works on large datasets A unpredictability fixing way has been established. Furthermore, afterward the consistency compute and stimulating reaching procedure have been recommended. A procedure algorithm for cluster decision creation is proposed on a large real dataset with huge number of prototypes. The mathematical instance regarding the range of the best journal for Excellence of Research in Australia Council has shown that our algorithm is valid and efficient.

\section{REFERENCES}

[1] J.Stegmann et al., "How to evaluate journal impact factors," Nature, vol.390, no. 6660, p. 550, 1997.

[2] Excellence in research for Australia (era). The Australian Research Council (ARC). [Online].Available: http://www.arc.gov.au/era/ R. Dobson, "Using data and experts to make the wrong decision," in Using Data to Improve Higher Education. Springer, 2014, pp. 229-242.

[3] J. Bollen, H. Van de Sompel, J. A. Smith, and R. Luce, "Toward alternative metrics of journal impact: A comparison of download and citation data," Information Processing \& Management, 2005

[4] E. Garfield, "The history and meaning of the journal impact factor, "JAMA: The Journal of the American Medical Association, vol. 295, no. 1,pp. 90-93, 2006.

[5] C. Bergstrom, J. West, and M. Wiseman, "The eigenfactor metrics," The Journal of Neuroscience, vol. 28, no. 45, pp. 11433 11 434, 2008.

[6] R. Rousseau, "Journal evaluation: Technical and practical issues," LibraryTrends, 2002.

[7] J. Stegmann and G. Grohmann, "Citation rates, knowledge export and international visibility of dermatology journals listed and not listed in journal citation reports," Scientometrics, 2001.

[8] J. Jamali, M. Salehi-Marzijarani, and S. M. T. Ayatollahi, "Factors affecting journalquality indicator in scopus in obstetrics and gynecology journals: a longitudinal study (1999-2013),"Act an Informatica Medical, 2014.

[9] N. Otsu, "A threshold selection method from gray-level histograms, "Systems, Man and Cybernetics, IEEE Transactions on, vol. 9, no. 1, pp.62-64.

[10] S Saravanan, V Venkatachalam, S Then Malligai "Optimization of SLA violation in cloud computing using artificial bee colony"2015, 1(3), 323 -327 ISSN: 2394-9260, pp:410-414. 